\title{
Homologous flare-CME events and their metric type II radio burst association
}

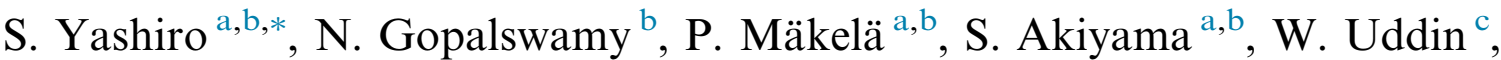 \\ A.K. Srivastava ${ }^{d}$, N.C. Joshi $^{j}$, R. Chandra ${ }^{\mathrm{e}}$, P.K. Manoharan ${ }^{\mathrm{f}}$, K. Mahalakshmi ${ }^{\mathrm{f}}$, \\ V.C. Dwivedi ${ }^{\mathrm{f}}$, R. Jain ${ }^{\mathrm{k}}$, A.K. Awasthi ${ }^{\mathrm{c}, \mathrm{g}}$, N.V. Nitta ${ }^{\text {h }}$, M.J. Aschwanden ${ }^{\mathrm{h}}$, \\ D.P. Choudhary ${ }^{\mathrm{i}}$ \\ ${ }^{a}$ The Catholic University of America, Washington, DC 20064, USA \\ ${ }^{\mathrm{b}}$ NASA Goddard Space Flight Center, Greenbelt, MD 20771, USA \\ ${ }^{\mathrm{c}}$ ARIES Nainital, Manora Peak, Nainital 263 129, India \\ ${ }^{\mathrm{d}}$ Department of Physics, Indian Institute of Technology (BHU), Varanasi 221 005, India \\ ${ }^{\mathrm{e}}$ Department of Physics, DSB Campus, Kumaun University, Nainital 263 002, India \\ ${ }^{\mathrm{f}}$ TIFR/NCRA Radio Astronomy Center, P. O. Box 8, Ootacamund 643 001, India \\ ${ }^{g}$ Physical Research Laboratory, Navrangapura, Ahmedabad 380 009, India \\ ${ }^{\mathrm{h}}$ Lockheed Martin Solar and Astrophysical Laboratory, Palo Alto, CA 94304, USA \\ ${ }^{\mathrm{i}}$ Department of Physics and Astronomy, California State University Northridge, Northridge, CA 91330, USA \\ ${ }^{\mathrm{j}}$ School of Space Research, Kyung Hee University, Yongin, Gyeonggi-Do 446-701, South Korea \\ ${ }^{\mathrm{k}}$ Kadi Sarva Vishwavidyalaya, Gandhinagar 382 015, Gujarat, India
}

Received 11 June 2013; received in revised form 5 June 2014; accepted 1 July 2014

Available online 14 July 2014

\begin{abstract}
Active region NOAA 11158 produced many flares during its disk passage. At least two of these flares can be considered as homologous: the C6.6 flare at 06:51 UT and C9.4 flare at 12:41 UT on February 14, 2011. Both flares occurred at the same location (eastern edge of the active region) and have a similar decay of the GOES soft X-ray light curve. The associated coronal mass ejections (CMEs) were slow ( 334 and $337 \mathrm{~km} / \mathrm{s})$ and of similar apparent widths $\left(43^{\circ}\right.$ and $\left.44^{\circ}\right)$, but they had different radio signatures. The second event was associated with a metric type II burst while the first one was not. The COR1 coronagraphs on board the STEREO spacecraft clearly show that the second CME propagated into the preceding CME that occurred 50 min before. These observations suggest that CME-CME interaction might be a key process in exciting the type II radio emission by slow CMEs.
\end{abstract}

(C) 2014 COSPAR. Published by Elsevier Ltd. All rights reserved.

Keywords: Flares; CMEs; Shocks; Radio emission

\section{Introduction}

Solar flares that occur at the same locations in the same active region within a certain time interval are called

\footnotetext{
* Corresponding author at: NASA Goddard Space Flight Center, Greenbelt, MD 20771, USA.

E-mail address: seiji.yashiro@nasa.gov (S. Yashiro).
}

recurrent flares. When the observed properties have many similarities in space and time, they are called homologous flares (Gaizauskas, 1982; Gaizauskas and Švestka, 1987). The homologous flares and the associated coronal mass ejections (CMEs) have been investigated to understand the energy buildup, the triggering mechanism, and the flare-CME relationship (e.g., Machado, 1985; Nitta and Hudson, 2001; Zhang and Wang, 2002; Moon et al., 2003; Chandra et al., 2011). 
There is a general consensus that type II radio bursts are a signature of shock waves propagating away from the Sun (Uchida, 1960; Nelson and Melrose, 1985; Gopalswamy et al., 2005). It has been established that shocks driven by CMEs are responsible for interplanetary type II bursts observed at decameter-hectometric (DH) wavelengths (Gopalswamy et al., 2001a) and kilometric wavelengths (Cane et al., 1987). However, the drivers of shocks associated with the metric (or coronal) type II radio bursts is still controversial: CME-driven shocks as in IP type II bursts (Cliver et al., 1999; Gopalswamy et al., 2009a; Cho et al., 2011) and flare blast waves (Vršnak et al., 1995; Khan and Aurass, 2002; Magdalenić et al., 2008) are the two possibilities.

Recent observations using the Solar TErrestrial RElations Observatory (STEREO) seem to indicate that even the coronal type II bursts are due to CMEs though there is a controversy over which part of the CMEs drive the shock, i.e., the CME nose (e.g., Gopalswamy et al., 2009a) or the CME flank (e.g., Liu et al., 2009). In order to shed additional light on this issue, we investigated the metric type II burst that was associated with an eruption in Active Region (AR) 11158. On 2011 February 14, seven eruptions occurred in AR 11158. Table 1 summarizes the 7 eruptions that occurred on February 14, 2011. At least two of them were homologous. The two eruptions were associated with C-class flares on the solar disk (labeled 1 and 2 in Table 1). The second C-class flare was associated with a metric type II radio burst and a slow CME. Six hours before the burst, the first C-class eruption occurred in AR 11158 but it did not produce a metric type II burst. The two eruptions had many similarities, but the occurrence of the metric type II burst was conspicuously different. In this paper we compare and contrast these two homologous flare-CME events.

\section{Radio bursts}

The homologous eruptions occurred at $\sim 7$ UT and $\sim 13$ UT on February 14, 2012. The San Vito Solar Observatory of the Radio Solar Telescope Network (RSTN) observed both events at the frequency range from $25 \mathrm{MHz}$ to $180 \mathrm{MHz}$. The detection threshold of radio emissions is always an issue when we investigate solar eruptions lacking the radio emissions. Fortunately, the detection issue is minimized because the same instrument was observing during both events. In the following, the flareCME event accompanied by the type II burst is referred to as radio-loud (RL), while the one lacking a type II is termed radio-quiet (RQ).

Fig. If is the radio dynamic spectrum for the second event. The white curve in the plots shows the soft X-ray flare intensity profile in the GOES long-wavelength band (1-8 $\mathrm{A}$ ) for reference. The second event was associated with a clear metric type II burst observed from 13:00 UT to 13:13 UT. Therefore this is a RL event.

Fig. 1a shows that no clear type II burst was associated with the first event. The radio emission at $\sim 80 \mathrm{MHz}$ at 07:06 UT (indicated by “?”) shows a short-duration feature which appears to drift in frequency. This feature could be a type II burst, a combination of type III bursts, or something else. The type II association of the first event may not be impossible. However we treat the first event as a RQ because the possible type II signature is so weak and uncertain compared to the second event.

\section{Flares}

In order to investigate the flare properties of the homologous events, we used EUV images obtained by the Atmospheric Imaging Assembly (AIA; Lemen et al., 2012) on board the Solar Dynamics Observatory (SDO; Pesnell et al., 2012). The magnetograms obtained by the Helioseismic and Magnetic Imager (HMI; Scherrer et al., 2012) on SDO were also used to investigate the magnetic configuration of AR 11158. Composite images of the HMI magnetograms and AIA $94 \AA$ EUV images have been created to investigate the flare location with respect to the AR magnetic field configuration. Fig. $1 \mathrm{~b}$ shows the composite image corresponding to the non-flaring period. Red and blue colors indicate positive and negative polarities of the magnetic field, respectively. Green color indicates the EUV intensity at $94 \AA$, indicating emission from a $6 \mathrm{MK}$ flare plasma.

AR 11158 was formed by two major bipoles, which emerged almost simultaneously on February 9, 2011. Both

Table 1

Seven eruptive events on 2011 Feb 14.

\begin{tabular}{|c|c|c|c|c|c|c|c|c|}
\hline \multirow[t]{2}{*}{ Label } & \multicolumn{3}{|c|}{ X-ray flare } & \multicolumn{2}{|c|}{ EUV wave } & \multicolumn{2}{|l|}{ CME } & \multirow[t]{2}{*}{ Type II } \\
\hline & Time & Intensity & Location & Time & Speed $^{\mathrm{a}}$ & Time & Speed $^{\mathrm{b}}$ & \\
\hline- & $02: 35$ & C1.6 & S21E04 & $02: 42$ & 523 & $02: 55$ & 264 & No \\
\hline $1 \mathrm{P}$ & 04:29 & C8.3 & S20W01 & $04: 44$ & $\sim 500$ & $04: 55$ & 312 & No \\
\hline 1 & $06: 51$ & C6.6 & S21E02 & $06: 56$ & 632 & 07:05 & 334 & No \\
\hline $2 \mathrm{P}$ & $11: 51$ & $\mathrm{C} 1.7$ & S21W01 & $12: 14$ & $\sim 500$ & $12: 15$ & 273 & No \\
\hline 2 & $12: 41$ & C9.4 & $\mathrm{S} 21 \mathrm{~W} 02$ & $12: 50$ & 661 & 13:05 & 337 & Yes \\
\hline- & $17: 20$ & M2.2 & S20W04 & $17: 28$ & 800 & $17: 35$ & 507 & Yes \\
\hline- & $19: 23$ & C6.6 & S20W05 & $19: 31$ & 495 & $19: 45$ & 355 & No \\
\hline
\end{tabular}

\footnotetext{
${ }^{\mathrm{a}}$ EUV wave speed in $\mathrm{km} / \mathrm{s}$.

${ }^{b} \mathrm{CME}$ speed in $\mathrm{km} / \mathrm{s}$.
} 

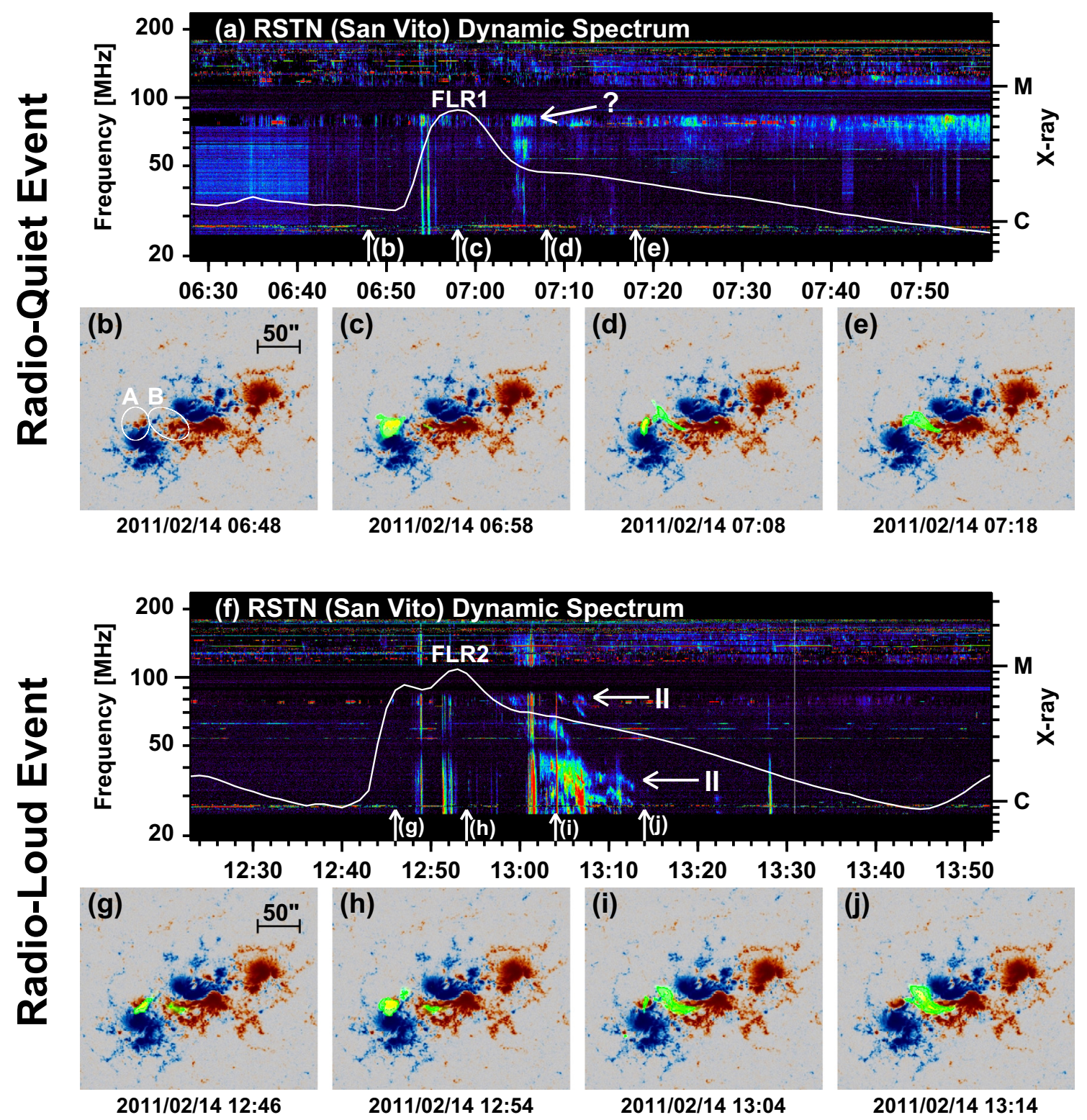

Fig. 1. (a, f) Radio dynamic spectrum observed by the San Vito Solar Observatory of the Radio Solar Telescope Network (RSTN). The observed frequency range is from 25 to $180 \mathrm{MHz}$. The white curve shows the GOES X-ray intensity in the long-wavelength band. The peak X-ray intensity was C6.6 for the first flare (FLR1) and C9.4 for the second flare (FLR2). (b-e, g-j) Magnetogram images observed by SDO/HMI. Red and blue colors correspond to positive and negative polarities. The overlaid green images were the SDO/AIA $94 \AA$ A observations, indicating the location of FLR1 and FLR2.

of the bipoles developed quickly, and the positive polarity of the SE bipole and the negative polarity of the NW bipole were attached on February 11 and formed the main neutral line of this AR. Many M- and X-class flares occurred at this main neutral line, but the homologous flares occurred at different neutral lines marked " $A$ " and " $B$ " in Fig. 1 b.

The X-ray flare of the first event (FLR1) started at 06:51 UT. The white curve in Fig. 1a shows the soft Xray intensity profile in the GOES $1-8 \AA$ band. Before FLR1, the X-ray intensity level was C1.2. FLR1 reached the maximum intensity of C6.6 level at 06:58 UT. The flare location was S21E02. The decay of FLR1 can be divided into two phases. The first one is the quick decay just after the peak. The X-ray intensity decreased to the half maximum in $5 \mathrm{~min}$. The second one is the long decay after 07:05 UT to the end of the flare. The X-ray intensity returned to the pre-flare level at around 07:40 UT ( $\sim 42$ min after the peak).

Fig. 1c is the AIA/HMI image taken at the peak time of FLR1, showing the flare kernel located at site-A. This flare kernel was compact $(\sim 2500 \mathrm{~km})$ and short-lived $(\sim 12 \mathrm{~min})$. Another part of the AR flared as the brightest location in the AIA $94 \AA$ images shifted to site-B during the decay phase (Fig. 1d and e).

The second flare (FLR2) started at 12:40 UT. The GOES X-ray curve shows two peaks at 12:46 UT and 
12:53 UT with intensity levels at C7.2 and C9.4, respectively. Fig. $1 \mathrm{~g}$ and $\mathrm{h}$ are the SDO/HMI-AIA composite images obtained at the times of the two peaks, showing that the AR brightened at the same location during the two peaks. The same location also brightened at the maximum of FLR1.

FLR2 also has a decay phase similar to that of FLR1. The X-ray intensity decreased to the half maximum in 6 min followed by a slow decrease beyond 13:00 UT. The $\mathrm{X}$-ray intensity returned to the pre-flare level at around 13:45 UT ( $\sim 52$ min after the peak). During the decay phase another part of the AR became excited as the brightest location in the AIA $94 \AA$ images is seen to shift to site-B (Fig. 1i and j).

The properties of FLR1 and FLR2 were very similar. Both flares were of C-class and the peak intensity of FLR2 was only $42 \%$ greater than that of FLR1 (C6.6 for FLR1 and C9.4 for FLR2). The brightest location at the flare peak was at site-A (see Fig. 1b) for both events. The flare plasmas at this location were short-lived. The X-ray intensity decayed to the half maximum in 5-6 min. On the other hand, the flare plasmas at site-B (see Fig. 1b) were long-lived. It took $40-50 \mathrm{~min}$ for the X-ray intensity to return to the pre-flare background level. One notable difference was that FLR2 had a double peak shown in the GOES $\mathrm{X}$-ray light-curve while FLR1 had a single peak.

\section{EUV waves}

Both the first and second event were associated with EUV waves (EWAV1 for the RQ and EWAV2 for the RL events). Fig. 2 shows AIA $193 \AA$ images for EWAV1 (top panels) and EWAV2 (bottom panels). Panels b-e and $\mathrm{g}-\mathrm{j}$ are running difference images to show the propaga- tion of the EUV waves. Both EUV waves originated from the site-A in AR 11158. Initially the wave fronts propagated only within a 90 degree cone opening toward NE (Fig. 2b and g) and spread to east and west (Fig. 2d and i). The speed of the eastern fronts was $632 \mathrm{~km} / \mathrm{s}$ for EWAV1 and $661 \mathrm{~km} / \mathrm{s}$ for EWAV2. The projection effects were corrected assuming that the wave fronts seen in Fig. 2 were located on the solar surface. These speeds are consistent with results of Nitta et al. (2013) who investigated 171 EUV waves. There was no significant difference in the EUV wave speeds between the RQ and RL event.

One notable difference was the brightness of the EUV wave front. Fig. $2 b$ and $g$ are the images taken at the peak time of the X-rays. At this time, EWAV1 (Fig. 2b) had clear and sharp front compared to the EWAV2 (Fig. 2g). The front of the EWAV2 became clear and was visible at the time of the type II burst (13:00 UT; 7 min after the flare peak). EWAV1 started to fade after the flare peak (Fig. 2b). Nine minutes after the flare peak (Fig. 2e), it is hard to identify the wave front in individual frames, but they can be seen in a movie.

\section{Coronal mass ejections}

The CMEs were observed in white-light by the inner coronagraph (COR1) of the Sun Earth Connection Coronal and Heliospheric Investigation (SECCHI; Howard et al., 2008) instrument onboard STEREO (Kaiser et al., 2008). The STEREO Ahead (STA) and Behind (STB) spacecraft were located $87^{\circ}$ ahead and $94^{\circ}$ behind of the Sun-Earth line, so the CMEs were observed from the side, i.e., the projection effects were minimized.

Fig. 3 shows white-light coronagraph images superimposed with EUVI $195 \AA$ images. The CME associated with

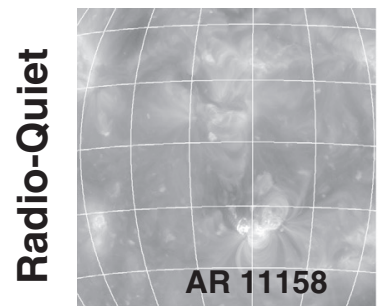

(a) 06:55

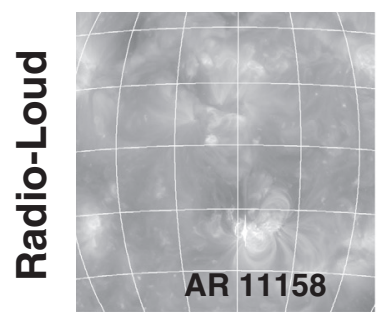

(f) 12:50

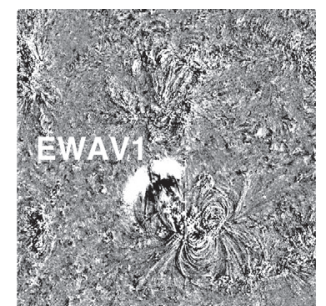

(b) $06: 58$

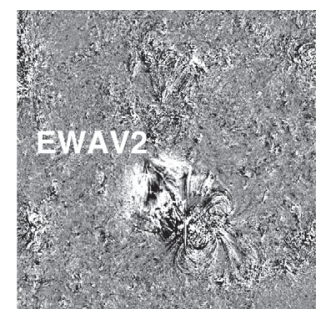

(g) $12: 53$

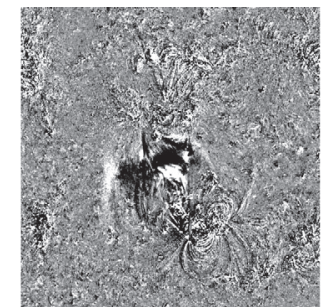

(c) 07:01

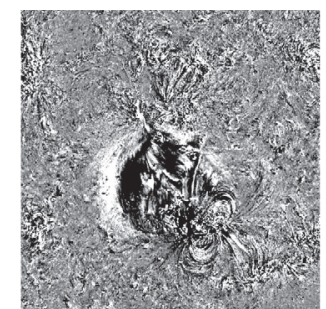

(h) $12: 56$

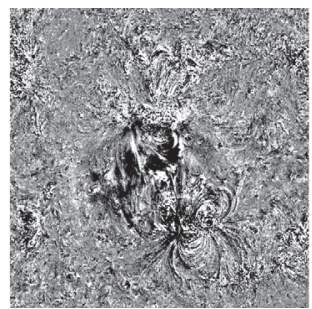

(d) 07:04

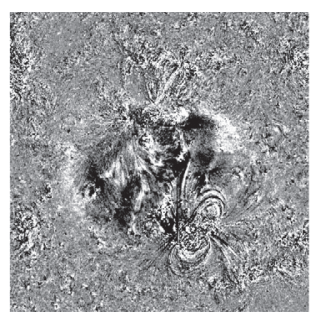

(i) $12: 59$

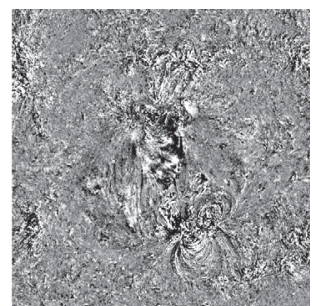

(e) 07:07

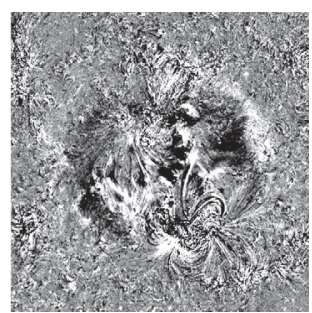

(j) $13: 02$

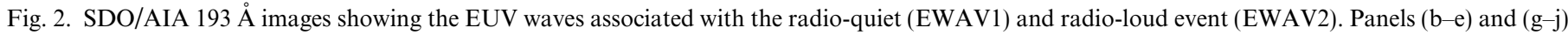

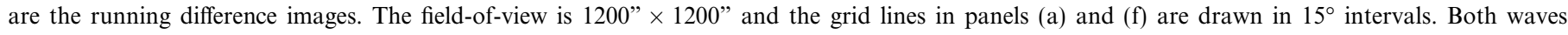

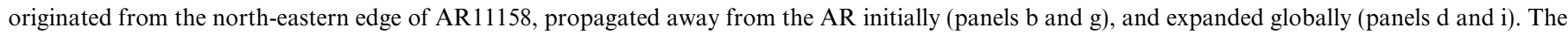
propagation speeds were $632 \mathrm{~km} / \mathrm{s}$ for EWAVE1 and $661 \mathrm{~km} / \mathrm{s}$ for EWAV2. 


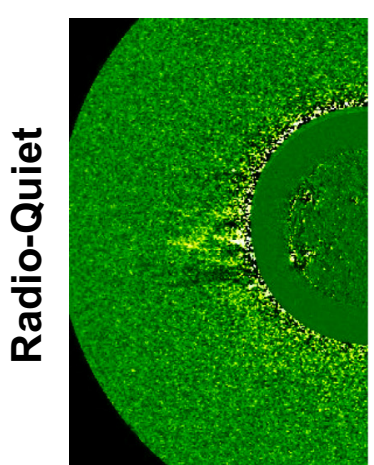

(a) 06:45

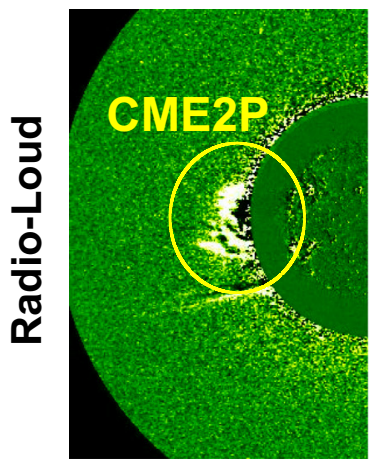

(f) $12: 35$

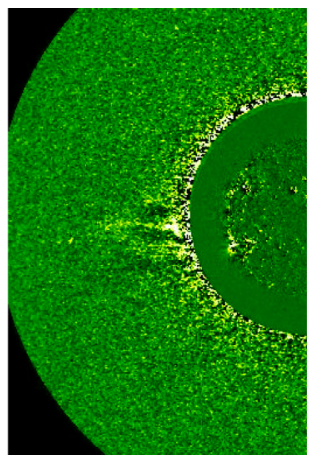

(b) $06: 55$

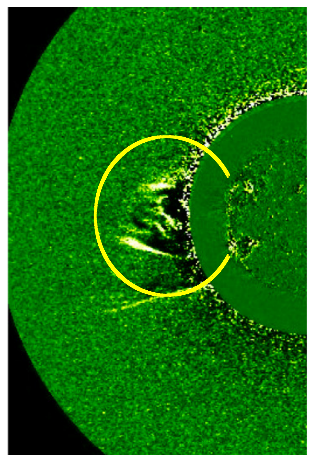

(g) $12: 45$

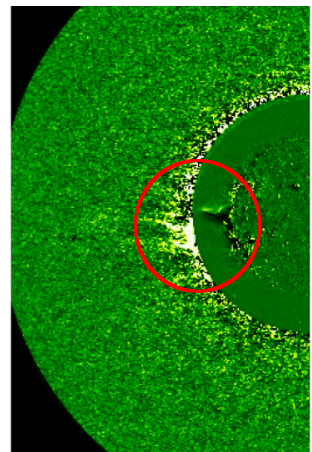

(c) 07:05

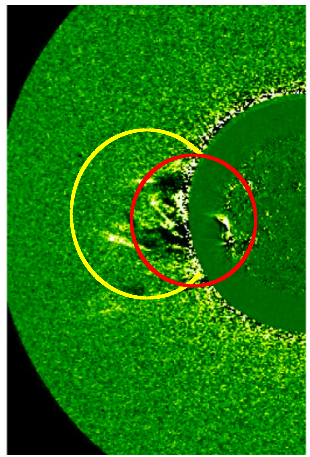

(h) $12: 55$

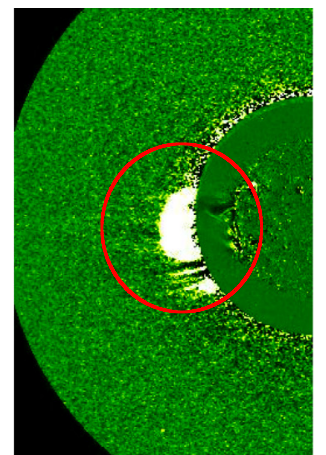

(d) 07:15

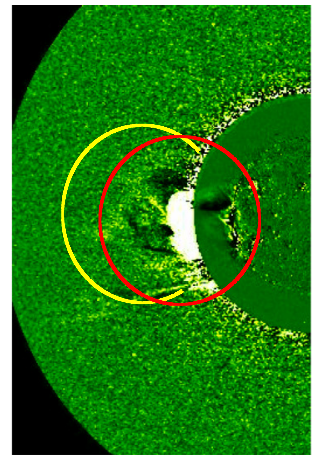

(i) $13: 05$

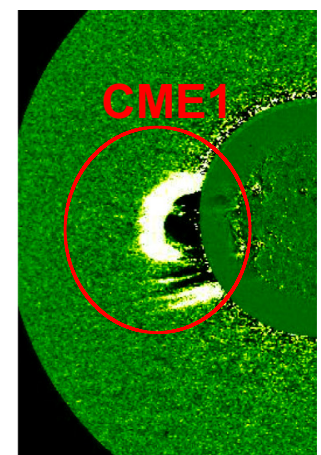

(e) 07:25

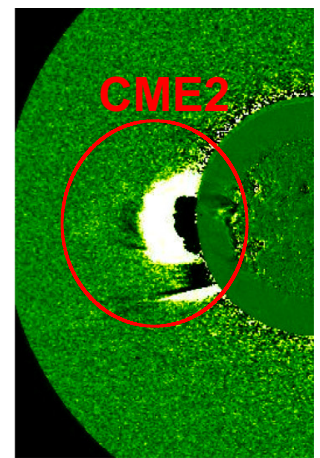

(j) $13: 15$

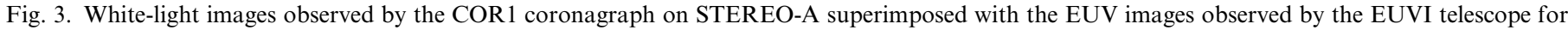

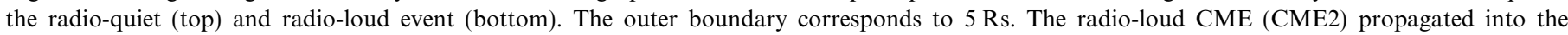

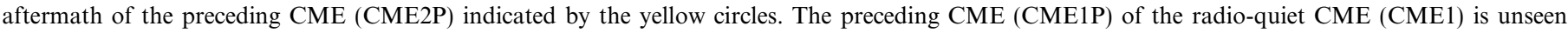
because it occurred $2 \mathrm{~h}$ before CME1.

the first event (CME1) appeared in the STA/COR1 field of view at 07:05 UT. The position angle of CME1, measured counterclockwise from the north in degrees, was $93^{\circ}$. The CME material extended from $67^{\circ}$ to $110^{\circ}$, giving an apparent width of $\sim 43^{\circ}$. The vertical black-and-white feature observed to the south of CME1 is a streamer deflection caused by the CME (Hundhausen, 1987; Sheeley et al., 2000). The CME associated with the second event (CME2) appeared at 13:05 UT (Fig. 3i), with its material extending from position angles $68^{\circ}$ to $112^{\circ}$ at 13:15 UT (Fig. 3j), hence its apparent width was $44^{\circ}$.

We carried out the height-time measurements for CME1 and CME2 (see Fig. 4). The CME heights are defined as distances from the center of the Sun to the nose of the CMEs. The average speeds in the COR1 field-ofview were 334 and $337 \mathrm{~km} / \mathrm{s}$, and both CMEs show deceleration. The second-order polynomial fit to the height-time data gives us acceleration of -74 and $-35 \mathrm{~m} / \mathrm{s}^{2}$ for CME1 and CME2, respectively. The initial speeds derived from the first two measurements were 483 and $471 \mathrm{~km} / \mathrm{s}$.

The CME properties were very similar in the RQ and RL event. The CME speeds were $334 \mathrm{~km} / \mathrm{s}$ for CME1 (RQ event) and $337 \mathrm{~km} / \mathrm{s}$ for CME2 (RL event). As we can see in Fig. 3e and j, the shape of both CMEs was also very similar. The apparent widths were $43^{\circ}$ for the CME1 and $44^{\circ}$ for CME2. We note that both CME1 and CME2 were less energetic compared to the average CMEs (Yashiro et al., 2004; Gopalswamy et al., 2009b).

\section{1. $C M E-C M E$ interaction}

There was no significant difference between the properties of CME1 and CME2, but the white-light coronagraph data indicate a major difference: CME1 propagated into the normal corona while CME2 propagated into the aftermath of a preceding CME (CME2P). Fig. 3f-i show CME2P marked by yellow circles. CME2P extended from $63^{\circ}$ to $108^{\circ}$ and it was widely overlapping with CME2.

The speed of CME2P was $273 \mathrm{~km} / \mathrm{s}$, so CME2 caught up with it. At 12:55 UT (Fig. 3h), the nose of CME2P was located at $2.5 \mathrm{Rs}$. A base difference image, which was obtained by subtracting the intensity of the pre-CME corona, shows that the material of CME2P extended down to the inner edge of the COR1 FOV (1.5 Rs). Therefore, the thickness of CME2P was at least 1 Rs. The gray shadow under the CME2P height-time plot in Fig. 4b represents the thickness of CME2P. We assume that the thickness was constant of $1 \mathrm{Rs}$. With this assumption, the first-order polynomial fit to the height-time data suggests that the CME-CME interaction started at 13:03:19 UT, which is 

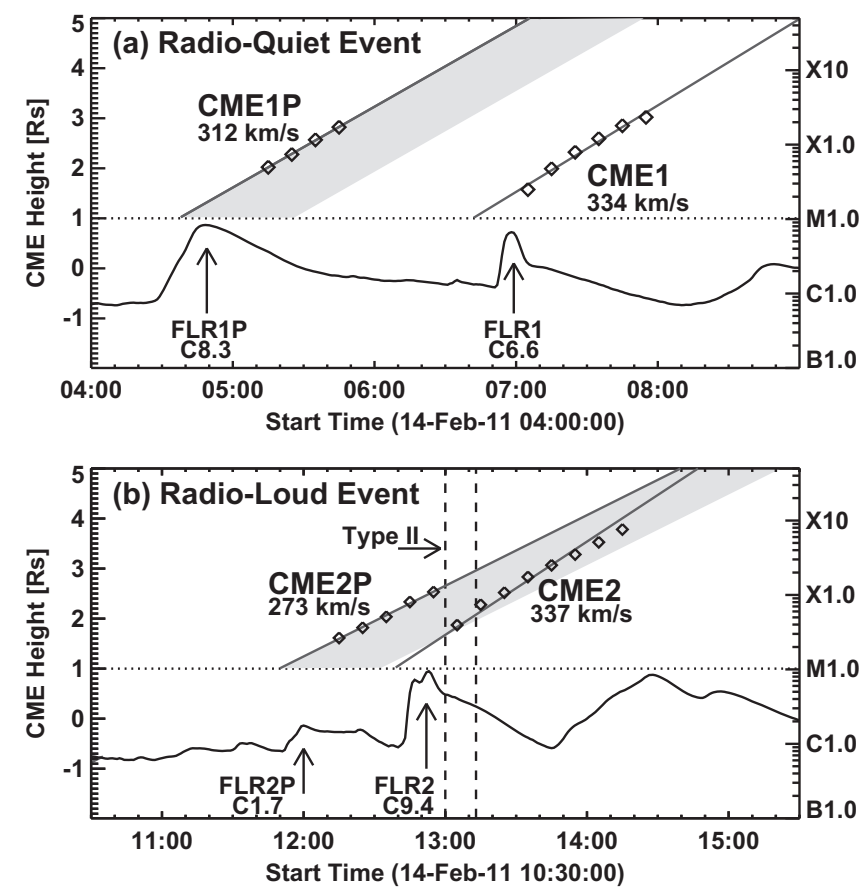

Fig. 4. CME height-time plots (diamonds) and GOES X-ray light curves for the (a) radio-quiet and (b) radio-loud event. The duration of the metric type II radio burst is indicated by the dashed lines. All flares noted in the plots occurred at the same location in AR 11158. The CME heights are measured from the center of the Sun in solar radii (Rs), i.e., the solar limb corresponds to $1 \mathrm{Rs}$. The shadows under CME1P and CME2P represent their thickness, showing that CME2 propagated into CME2P around the onset of type II burst.

close to the start time of the type II (13:00 UT). If we apply the second-order polynomial fit, we obtain 13:03:56 UT. The start time of the CME-CME interaction would be earlier because the CME2P could be thicker than 1 Rs in reality.

CME1 also had a preceding CME (CME1P) erupting from the same region. CME1P was associated with a C8.3 flare that started at 04:29 UT (marked as FLR1P in Fig. 4). The speed of CME1P was $312 \mathrm{~km} / \mathrm{s}$. The measured thickness of CME1P was approximately 1.3 Rs at 05:45 UT. As we see in Fig. 4a, the height-time plot of CME1 does not cross the gray bar of CME1P. Even if we assume the CME thickness to be double (2.6 Rs), CME1 could not catch up with CME1P. Because the speed difference was so small and CME1P erupted $2 \mathrm{~h}$ before the CME1, no CMECME interaction occurred in the COR1 FOV.

Fig. 3i shows that CME2 propagated into the material of CME2P. No CME-CME interaction occurs if CMEs are at different locations along the same light-of-sight. CME2P was associated with a C1.7 flare (FLR2P) starting 50 min earlier than FLR2. The location of FLR2P was the same as that of FLR2. CME2P was associated with a faint EUV wave (EWAV2P), which originated from the eastern edge of AR 11158 and propagated to the NE direction, similar to EWAV2. The source region of CME2P was the same as that of CME2. So if all CMEs erupted in the same direction, CME-CME interaction is highly likely.
In addition to the four CMEs (CME1P, CME1, CME2P, and CME2) we considered above, three other CMEs erupted from the same location on the same day at 02:55 UT, 17:35 UT, and 19:45 UT in the COR1 FOV (see Table 1). A CME seen at 06:25 UT in the COR1 data is not listed in Table 1 because the CME originated from different location (S28W22). All seven CMEs originated from disk center. Although the seven CMEs did not look quite the same, their trajectories were similar as inferred from the COR 1 images. If the launch angle of the seven CMEs were different, the CMEs should appear different in the LASCO data. The two CMEs at 13:05 UT and 17:35 UT were observed by LASCO. The CME material appeared on both east and west sides, so these two CMEs were launched radially. For the remaining five CMEs, we could not identify clear LASCO counterparts. It is likely that the five CMEs erupted radially with smaller angular extents since it is difficult to observe Earth-directed CMEs owing to the nature of the coronagraph observations (Yashiro et al., 2005). Therefore, we did not find any indication that any of the five CMEs erupted non-radially. It is reasonable to assume that all CMEs erupted to the same direction.

\section{Discussion and conclusions}

Gopalswamy et al. (2001c) discovered that interplanetary type II radio emission was enhanced by the CME$\mathrm{CME}$ interaction. Using radio direction finding techniques, Martínez-Oliveros et al. (2012) confirmed that the source position of the interplanetary type II radio emission on 2010 August 1 was in good agreement with the CMECME interaction region. Gopalswamy et al. (2002) reported an interplanetary type II radio burst that was solely attributable to a CME-CME interaction. Our finding is similar to this case but it happened closer to the Sun in the metric corona.

MHD shock waves form when the CME speed exceeds the Alfvén speed of the medium. Gopalswamy et al. (2001b, 2012) suggested that Alfvén speed profile as a function of the heliocentric distance has a local minimum $(\sim 375 \mathrm{~km} / \mathrm{s})$ at around $1.5 \mathrm{Rs}$. Around this region, shocks can be formed easily. The metric type II bursts typically start when CMEs reach this height (Gopalswamy et al., 2009a, 2013). In our case the type II radio emission started when the CME reached 1.74 Rs. The speed around this height was $483 \mathrm{~km} / \mathrm{s}$ for the RQ and $471 \mathrm{~km} / \mathrm{s}$ for the RL CMEs. Both CMEs had enough speed to produce a shock but their radio properties were different. The material of the preceding CME might have changed the local minimum of the Alfvén speed profile to be deeper compared to the normal corona. Although the CME speeds were very similar in the RQ and RL events, the Alfvén Mach number might have been different due to the preceding CME.

Wagner and MacQueen (1983) proposed that metric type II radio emission occurs when flare blast waves overtake the preceding CME. Our result might support their 
idea, but the flare blast wave was not seen in the COR1 data so it is unclear when the flare blast wave overtook the preceding CME. The strong type II radio emission occurred when the primary CME started catching up with the preceding CME (see Fig. $3 i$ and Fig. 4b). This observation supports the CME-CME interaction scenario.

Another possibility is that type II radio bursts are produced by the CME-streamer interaction. The streamer deflection is a signature of (shock) waves driven by a CME (Hundhausen, 1987; Sheeley et al., 2000). Liu et al. (2009) reported that the metric type II burst and streamer deflection associated with 2007 December 31 CME occurred simultaneously. They concluded that the streamer deflection that occurred at the flank of the CME was the source of the type II radio burst. In our case a streamer defection associated with the RL event was first observed at 13:05 UT which was close to time of the strong type II emission ( 13:04 UT; see Fig. 1f). The observation is consistent with the CME-streamer interaction scenario. On the other hand, the RQ CME also caused a streamer deflection (Fig. 3e). Obviously a streamer deflection by itself is not a necessary condition to excite a type II radio burst. It should be noted that a streamer deflection occurred in association with the CME that preceded the RL CME (Fig. 3f). The preceding CME might have changed the condition of the streamer to enhance the shock driven by the RL CME.

We investigated two homologous eruptions, one accompanied by a type II radio burst and the other not. Since the homologous events occurred just $6 \mathrm{~h}$ apart, the magnetic field environment around the flare site can be thought to be the same during the two eruptions. The homologous eruptions had many similarities, e.g., the flare location within the active region (Fig. $1 \mathrm{~b}-\mathrm{d}$ and $\mathrm{g}-\mathrm{j}$ ), the peak $\mathrm{X}$ ray intensity, the X-ray light curve (Fig. 1a and f), EUV wave speed (Fig. 2), the CME apparent width (Fig. 3) and CME apparent speed (Fig. 4). We did not find significant differences in the usual parameters that characterize flares and CMEs. We did find a major difference in the coronal environment into which the CMEs were ejected. The RL event had a preceding CME that erupted $50 \mathrm{~min}$ earlier while the RQ event did not have one in such quick succession. Therefore, we conclude that preconditioning must be a key aspect as to why the homologous events had different radio properties.

\section{Acknowledgements}

This study was conducted as a part of the Indo-US Science and Technology Forum's Joint Center on Solar Eruptive Events. Part of the work is also supported by NASA LWS TR\&T program.

\section{References}

Cane, H.V., Sheeley Jr., N.R., Howard, R.A., 1987. Energetic interplanetary shocks, radio emission, and coronal mass ejections. J. Geophys. Res. 92 (1), 9869-9874.
Chandra, R., Schmieder, B., Mandrini, C.H., Démoulin, P., Pariat, E., Török, T., Uddin, W., 2011. Homologous flares and magnetic field topology in active region NOAA 10501 on 20 November 2003. Sol. Phys. 269, 83-104.

Cho, K.-S., Bong, S.-C., Moon, Y.-J., Shanmugaraju, A., Kwon, R.-Y., Park, Y.D., 2011. Relationship between multiple type II solar radio bursts and CME observed by STEREO/SECCHI. Astron. Astrophys. 530 (A16).

Cliver, E.W., Webb, D.F., Howard, R.A., 1999. On the origin of solar metric type II bursts. Sol. Phys. 187 (1), 89-114.

Gaizauskas, V., 1982. The relation of solar flares to the evolution and proper motions of magnetic fields. Adv. Space Res. 2, 11-30.

Gaizauskas, V., Švestka, Z., 1987. Highlights of the flare build-up study, Sol. Phys. 114, 389-398.

Gopalswamy, N., Yashiro, S., Kaiser, M.L., Howard, R.A., Bougeret, J.L., 2001a. Characteristics of coronal mass ejections associated with long-wavelength type II radio bursts. J. Geophys. Res. 106, 29219 29230.

Gopalswamy, N., Lara, A., Kaiser, M.L., Bougeret, J.-L., 2001b. Nearsun and near-earth manifestations of solar eruptions. J. Geophys. Res. 106 (A11), 25261-25278.

Gopalswamy, N., Yashiro, S., Kaiser, M.L., Howard, R.A., Bougeret, J.L., 2001c. Radio signatures of coronal mass ejection interaction: coronal mass ejection cannibalism? Astrophys. J. 548 (1), L91-L94.

Gopalswamy, N., Yashiro, S., Kaiser, M.L., Howard, R.A., Bougeret, J.L., 2002. Interplanetary radio emission due to interaction between two coronal mass ejections. Geophys. Res. Lett. 29 (8), 106-1, CiteID 1265.

Gopalswamy, N., Aguilar-Rodriguez, E., Yashiro, S., Nunes, S., Kaiser, M.L., Howard, R.A., 2005. Type II radio bursts and energetic solar eruptions. J. Geophys. Res. 110 (A12), CiteID A12S07.

Gopalswamy, N., Thompson, W.T., Davila, J.M., Kaiser, M.L., Yashiro, S., Mäkelä, P., Michalek, G., Bougeret, J.-L., Howard, R.A., 2009a. Solar Phys. 259 (1-2), 227-254.

Gopalswamy, N., Yashiro, S., Michalek, G., Stenborg, G., Vourlidas, A., Freeland, S., Howard, R., 2009b. Earth Moon Planet. 104 (1-4), 295 313.

Gopalswamy, N., Xie, H., Yashiro, S., Akiyama, S., Mäkelä, P., Usoskin, I.G., 2012. Properties of ground level enhancement events and the associated solar eruptions during solar cycle 23. Space Sci. Rev. 171 (1-4), 23-60

Gopalswamy, N. et al., 2013. Height of shock formation in the solar corona inferred from observations of type II radio bursts and coronal mass ejections. Adv. Space Res. 51, 1981-1989.

Howard, R.A. et al., 2008. Sun earth connection coronal and heliospheric investigation (SECCHI). Space Sci. Rev. 136, 67-115.

Hundhausen, A.J., 1987. The origin and propagation of coronal mass ejections, sixth international solar wind conference. In: Pizzo, V.J., Holzer, T., Sime, D.G. (Eds.), NCAR Technical Note NCAR/TN306+Proc, 2, p. 181.

Kaiser, M.L., Kucera, T.A., Davila, J.M., St. Cyr, O.C., Guhathakurta, M., Christian, E., 2008. The STEREO mission: an introduction. Space Sci. Rev. 136, 5-16.

Khan, J.I., Aurass, H., 2002. X-ray observations of a large-scale solar coronal shock wave. Astron. Astrophys. 383, 1018-1031.

Lemen, J.R. et al., 2012. The atmospheric imaging assembly (AIA) on the solar dynamics observatory (SDO). Sol. Phys. 275, 17-40.

Liu, Y., Luhmann, J.G., Bale, S.D., Lin, R.P., 2009. Relationship between a coronal mass ejection-driven shock and a coronal metric type II burst. Astrophys. J. Lett. 691, L151.

Machado, M.E., 1985. An evidence of flare energy buildup and release related to magnetic shear and reconnection. Sol. Phys. 99, 159-166.

Magdalenić, J., Vršnak, B., Pohjolainen, S., Temmer, M., Aurass, H., Lehtinen, N.J., 2008. A flare-generated shock during a coronal mass ejection on 24 December. Sol. Phys. 253 (1-2), 305-317.

Martínez-Oliveros, J.C., Raftery, C.L., Bain, H.M., Liu, Y., Krupar, V., Bale, S., Krucker, S., 2012. The 2010 august 1 type II burst: a CME$\mathrm{CME}$ interaction and its radio and white-light manifestations. Astrophys. J. 748, 66. 
Moon, Y.-J., Choe, G.S., Wang, H., Park, Y.D., Cheng, C.Z., 2003. Relationship between CME kinematics and flare strength. J. Korean Astron. Soc. 36, 61-66.

Nelson, G.J., Melrose, D.B., 1985. Type II bursts. Solar Radio Physics, Studies of Emission from the Sun at Metre Wavelengths. Cambridge University Press, pp. 333-359.

Nitta, N.V., Hudson, H.S., 2001. Recurrent flare/CME events from an emerging flux region. Geophys. Res. Lett. 28, 3801-3804.

Nitta, N.V., Schrijver, C.J., Title, A.M., Liu, W., 2013. Large-scale coronal propagating fronts in solar eruptions as observed by the atmospheric imaging assembly on board the solar dynamics observatory — an ensemble study. Astrophys. J. 776, 13.

Pesnell, W.D., Thompson, B.J., Chamberlin, P.C., 2012. The solar dynamics observatory (SDO). Sol. Phys. 275, 3-15.

Scherrer, P.H. et al., 2012. The helioseismic and magnetic imager (HMI) investigation for the solar dynamics observatory (SDO). Sol. Phys. $275,207-227$.

Sheeley, N.R., Hakala, W.N., Wang, Y.-M., 2000. Detection of coronal mass ejection associated shock waves in the outer corona. J. Geophys. Res. 105 (A3), 5081-5092.
Uchida, Y., 1960. On the exciters of type II and type III solar radio bursts. Publ. Astron. Soc. Jpn 12, 376.

Vršnak, B., Ruzdjak, V., Zlobec, P., Aurass, H., 1995. Ignition of MHD shocks associated with solar flares. Sol. Phys. 158 (2), 331-351.

Wagner, W.J., MacQueen, R.M., 1983. The excitation of type II radio bursts in the corona. Astron. Astrophys. 120, 136-138.

Yashiro, S., Gopalswamy, N., Michalek, G., St. Cyr, O.C., Plunkett, S.P., Rich, N.B., Howard, R.A., 2004. A catalog of white light coronal mass ejections observed by the SOHO spacecraft. J. Geophys. Res. 109 (A7), CiteID A07105.

Yashiro, S., Gopalswamy, N., Akiyama, S., Michalek, G., Howard, R.A., 2005. Visibility of coronal mass ejections as a function of flare location and intensity. J. Geophys. Res.: Space Phys. 110 (A12), CiteID A12S05.

Zhang, J., Wang, J., 2002. Are homologous flare-coronal mass ejection events triggered by moving magnetic features? Astrophys. J. 566, L117-L120. 